\title{
The Association Between Phosphorylated Neurofilament Heavy Chain (pNF-H) and Small Fiber Neuropathy (SFN) in Patients with Impaired Glucose Tolerance
}

\author{
Yu-peng Li (D) Z Zhong-qing Yan · Li-ping Han · Ai-li Yin • \\ Jin-yong Xu • Ya-ran Zhai · Sai Hao $\cdot$ Lin Zhang $\cdot$ Yun Xie
}

Received: July 26, 2019 / Published online: October 30, 2019

(C) The Author(s) 2019

\begin{abstract}
Introduction: Small fiber neuropathy (SFN)the early stage of diabetic peripheral neuropathy (DPN)-progresses gradually and is difficult to diagnose using neurophysiological tests. To facilitate the early diagnosis of SFN, biomarkers for SFN must be identified. The purpose of this study was to investigate the characteristics of SFN in prediabetic patients and the relationship between pNF-H and SFN.
\end{abstract}

Enhanced Digital Features To view enhanced digital features for this article go to https://doi.org/10.6084/ m9.figshare.9929315.

Y. Li (ه) - Z. Yan · L. Han · A. Yin · J. Xu · Y. Zhai ·

S. Hao $\cdot$ L. Zhang $\cdot$ Y. Xie $(\varangle)$

Tianjin Medical University Metabolic Diseases

Hospital \& Tianjin Medical University Chu Hsien-I

Memorial Hospital, Tianjin, China

Y. Li

e-mail: dr_liyupeng@126.com

Y. Xie

e-mail: xieyuntj@126.com

Y. Li · Z. Yan · L. Han · A. Yin · J. Xu · Y. Zhai ·

S. Hao $\cdot$ L. Zhang $\cdot$ Y. Xie

Tianjin Institute of Endocrinology, Tianjin, China

Y. Li · Z. Yan · L. Han · A. Yin · J. Xu · Y. Zhai .

S. Hao $\cdot$ L. Zhang · Y. Xie

NHC Key Laboratory of Hormones and

Development (Tianjin Medical University), Tianjin

Key Laboratory of Metabolic Diseases, Tianjin,

China
Methods: 44 IGT patients (inpatients and outpatients) were selected at random. 33 healthy subjects served as controls. Data on clinical characteristics and laboratory parameters were collected. Quantitative sensory testing (QST), electromyography (EMG), and Sudoscan were performed, and pNF-H was measured by ELISA. Results: 24 of the 44 patients with impaired glucose tolerance (IGT) were diagnosed with SFN according to the modified Toronto Diabetic Neuropathy Expert Group consensus criteria. The thermal sensory thresholds of the IGT-SFN group were significantly different from those of the CTRL group $(p<0.05)$, except for the heat pain threshold. The sensory nerve action potential (SNAP) of the sural nerve was 12.39 in the IGT-SFN group, which was significantly lower than those in the other groups. No significant difference in nerve conduction velocity (NCV) was observed among the three groups. The electrochemical skin conductance (ESC) in the IGT-SFN group was $69.78 \pm 14.03$ uS, which was significantly lower than that in the CTRL group. The pNF-H in the IGT-SFN group was $170.6(140.0,223.6) \mathrm{pg} / \mathrm{ml}$, which was significantly higher than those in the CTRL and IGTnon-SFN groups (76.55 and $64.7 \mathrm{pg} / \mathrm{ml}$, respectively). Multivariate regression analysis demonstrated that $\mathrm{pNF}-\mathrm{H}$ and $2 \mathrm{~h}$ plasma glucose were independently correlated with SFN; the ORs $(95 \%$ CI) were $1.429(1.315,1.924)$ and 2.375 $(1.157,4.837)$, respectively. 
Conclusions: Serum pNF-H may be associated with SFN in IGT patients, and serum pNF-H could therefore serve as a sensitive biomarker for the detection of SFN.

Keywords: Diabetic peripheral neuropathy; Nerve conduction; pNF-H; Quantitative sensory testing; Sensory nerve function; Small fiber neuropathy

\section{Key Summary Points}

Small fiber neuropathy (SFN) is considered the 'microalbuminuric' equivalent of diabetic peripheral neuropathy (DPN), and is often overlooked by physicians.

Early detection of SFN is critical for the prevention and treatment of DPN.

Diagnosis of SFN is complicated and time consuming. Up to now, no serum-based indicator that could be used for the specific detection of SFN had been identified.

Phosphorylation of neurofilament heavy chain (pNF-H) is crucial to axonal function and structure, and pNF-H could be released into blood during axonal damage. Axonal damage is considered to be indicative of early-stage SFN.

We hypothesize that pNF-H is closely related to SFN.

Our study selected patients with impaired glucose tolerance (IGT) as research subjects. The neurological complications of such patients have previously received little attention from researchers.

Our study demonstrated the general characteristics, biochemistry, autonomic nerve function, neurophysiology, and thermal thresholds of prediabetic patients with SFN.

Our study revealed a close relationship between pNF-H and SFN.

\section{INTRODUCTION}

Up to $50 \%$ of all diabetic patients experience neurological complications, although nearly half of those are asymptomatic [1, 2]. Diabetic peripheral neuropathy (DPN) is the most common complication. DPN is a gradually progressive neuropathy with a silent onset, and is often not diagnosed by specialists until it is well advanced. Small and large fiber nerves may be involved in DPN. Large fiber nerve damage is associated with paresthesia, sensory loss, and muscle weakness, while small fiber nerve damage often causes pain, anesthesia, foot ulcers, and autonomic symptoms [3]. Research indicates that DPN, including small fiber neuropathy (SFN) [4], may occur in patients with impaired glucose tolerance (IGT) [5, 6]. Abnormalities in the structure and function of small fiber nerves precede those in large fiber nerves in diabetes [7]. SFN may be considered the 'microalbuminuric' equivalent of diabetic sensorimotor neuropathy (DSPN) [7]. Early recognition of SFN facilitates early treatment and delays the progression of DPN.

Neurofilaments (NFs) are important skeletal proteins that are exclusively expressed in axons and play an essential role in maintaining neuronal tensile strength and the integrity of axons. NF-H, a heavy chain of three NF subunits, contributes to the formation of cross bridges and interactions with microtubules, microfilaments, etc. Phosphorylation of NFs can influence functional status. Phosphorylation of NF-H is essential for regulating axonal caliber and interfilament spacing, and it mediates interactions with microtubules and other structural elements. Compared to other NFs, phosphorylated neurofilament heavy chain (pNF-H) is more stable in the blood and less likely to be degraded, so it can be easily and consistently detected in the laboratory setting. pNF-H was proposed as a potential biomarker of DPN in a cross-sectional study [8]. Evidence indicates a close relationship between SFN and axonal damage $[9,10]$. Axonal damage is considered indicative of early-stage SFN [11]. Electrically stimulated axon reflexes that may indicate dysfunction in cutaneous $\mathrm{C}$ fibers are 
diminished in SFN. Small nerve fibers are unmyelinated or thinly myelinated fibers that are more likely to be affected by the surrounding environment. As NFs are major elements of the axon, we hypothesized that NF subunits, especially pNF-H, could be released into the blood during axonal damage. This study investigated whether pNF-H is an indicator of earlystage SFN in prediabetic IGT patients.

\section{METHODS}

\section{Study Population and Design}

This study was conducted at the Tianjin Medical University Metabolic Diseases Hospital and the Tianjin Institute of Endocrinology (Tianjin, China) from June 2017 to September 2018. This research was conducted in accordance with the Declaration of Helsinki. The study protocol was approved by the Ethics Committee of Tianjin Medical University Metabolic Diseases Hospital. All study participants provided informed consent. The study included 44 IGT patients (inpatients and outpatients) who were selected at random. A total of 33 normal subjects (healthy volunteers) were recruited to form the control (CTRL) group. IGT was diagnosed according to the standards defined by the American Diabetes Association (ADA) in 2012. All study participants were between 18 and 75 years of age. Exclusion criteria included a previous diagnosis of or treatment for DPN, sequelae of cerebral infarction, Alzheimer's disease, Parkinson's disease, peripheral vascular disease, an active foot ulcer, renal dysfunction (glomerular filtration rate, $G F R<60 \mathrm{~mL} / \mathrm{min} / 1.73 \mathrm{~m}^{2}$ ), secondary causes of DPN (including lumbar disease and alcoholic polyneuropathy), or the use of drugs that could affect sweat gland activity.

\section{Patient Clinical Characteristics}

The clinical characteristics, personal histories, and physical characteristics of the patients were recorded. Pinprick, temperature, vibration perception, pressure sensation, and ankle reflex tests were applied. The appearance of the feet and any symptoms (including burning, numbness, tingling, fatigue, cramping, or pain in the legs) were recorded. The Michigan Neuropathy Screening Instrument (MNSI; parts A and B) [12] and the Toronto Clinical Neuropathy Score (TCNS) were used to evaluate DPN.

\section{Quantitative Sensory Testing (QST)}

All subjects were trained in the QST procedure. A thermal sensory analyzer (TSA-2001; Medoc, Ramat Yishai, Israel) was used to determine warm and cool sensation thresholds as well as heat pain and cold pain thresholds using the limits method (increase the temperature in $1{ }^{\circ} \mathrm{C} / \mathrm{s}$ increments starting at $32^{\circ} \mathrm{C}$, repeat four times to assess warm and cool sensation thresholds, and repeat three times to assess heat pain and cold pain thresholds). Normal thresholds were defined according to the ageand sex-matched normative values obtained in our hospital. Sensory abnormality was defined as a response outside the normal range for at least two (both feet) of the four thermal sensation/pain thresholds.

\section{Electromyography (EMG)}

All nerve stimulation, including stimulation of the common peroneal and tibial motor nerves and stimulation of the peroneal and sural sensory nerves in both limbs, was performed with an EMG instrument (NDI-094C; Shanghai Haishen Medical Electronic Instrument Co. Ltd., Shanghai, China). Abnormalities were defined as observed values that exceeded the reference ranges established in our laboratory. All study participants were also tested using the NC-stat DPNCheck device (NeuroMetrix Inc., Waltham, MA, USA). An abnormal nerve conduction velocity (NCV) was considered indicative of large fiber neuropathy; all such cases were excluded.

\section{Sudoscan Results}

Tests were performed on all participants using a Sudoscan device (Impeto Medical, Paris, France). Electrochemical skin conductance (ESC) values were recorded while a low-voltage 
$(<4 \mathrm{~V})$ electrical current was applied. The mean of the left and right ESC values was used in the statistical analysis.

\section{Diagnosis of SFN}

SFN was diagnosed according to the Diabetic Neuropathy Study Group of the EASD (NEURODIAB) guidelines and the modified Toronto Diabetic Neuropathy Expert Group consensus [13]. SFN was defined as the presence of lengthdependent symptoms, clinical signs of small fiber damage [spontaneous pain (burning, sharp), evoked pain (allodynia, hyperalgesia), pruritus (alloknesis, hypernesis), paraesthesias, dysesthesias, and hypoesthesia (thermal and pinprick)], a normal sural nerve conduction (NC) study, and abnormal QST thermal thresholds in the dorsal part of the foot.

\section{Laboratory Parameters and pNF-H Measurements}

Blood samples were collected from all participants after overnight fasting. Fasting plasma glucose (FPG) and postprandial $2 \mathrm{~h}$ plasma glucose (2hPG), total cholesterol (TC), triglyceride (TG), high-density lipoprotein cholesterol (HDL-c), low-density lipoprotein cholesterol (LDL-c), blood urea nitrogen (BUN), serum creatinine $(\mathrm{Cr})$, and urinary creatinine concentration were analyzed using an automatic analyzer (model 7600; Hitachi, Tokyo, Japan) using an enzymatic assay. Glycosylated hemoglobin (HbA1c) was evaluated by high-pressure liquid chromatography (HLC-723G7; Tosoh Corporation, Tokyo, Japan). The urinary albumin/creatinine ratio (ACR) was calculated as the albumin level $(\mathrm{mg}$ ) divided by the creatinine level $(\mathrm{g})$. Serum pNF-H levels were analyzed using an ELISA kit (Human Phosphorylated Neurofilament H; BioVendor, Modrice, Czech Republic).

\section{Statistical Analysis}

Statistical analyses were performed using the SPSS software (version 24.0; SPSS Inc., Chicago, IL, USA). Normally distributed continuous variables are expressed in this work as mean (standard deviation); non-normally distributed variables are presented as median $(25 \%, 75 \%$ quartiles). One-way ANOVA with Student-Newman-Keuls (SNK) post hoc testing was used to assess the quantitative data. Correlations between variables were estimated by Spearman's correlation analysis. A two-tailed $p$ value $<0.05$ was considered to indicate statistical significance. Binary logistic regression using the forward stepwise method was performed to analyze independent related factors. All variables included in the regression were significant $(p<0.05)$ or showed a trend toward significance $(p<0.10)$.

\section{RESULTS}

\section{General Clinical Characteristics}

Among 61 IGT patients, 17 were excluded due to large fiber neuropathy. A total of 24 participants were diagnosed with SFN (not combined with large fiber neuropathy) according to the diagnostic criteria mentioned above (Table 1). No statistical difference was observed among the CTRL, IGT-non-SFN, and IGT-SFN groups in gender, BMI, waist-to-hip ratio (WHR), TG, TC, LDL-c, HDL-c, Cr, BUN, or ACR. The average age of the IGT-SFN group was higher than those of the other groups. HbA1c, FPG, and 2hPG were significantly higher in the IGT-SFN group than in the CTRL and IGT-non-SFN groups. There was a significant difference in $2 \mathrm{hPG}$ between the IGT-SFN and CTRL groups (Table 2).

The IGT-SFN group had a significantly higher warm sensation threshold and significantly lower cool and cold pain thresholds than the CTRL group $(p<0.05)$, as well as a significantly higher warm sensation threshold than the IGT-non-SFN group. No significant difference in heat pain threshold was observed among the three groups (Table 3 ).

Serum pNF-H (170.6 (140.0, 223.6) pg/ml) levels were significantly elevated in the IGT-SFN group $(p<0.0001)$. No significant difference was observed between the CTRL group $(76.55 \pm 28.08 \mathrm{pg} / \mathrm{ml})$ and IGT-non-SFN group $(64.7 \pm 18.73 \mathrm{pg} / \mathrm{ml})$ (Fig. 1).

There was no significant difference among the three groups in the SNCVs of the sural, 
Table 1 Inclusion and exclusion criteria for study participants

\begin{tabular}{|c|c|}
\hline Inclusion criteria & Exclusion criteria \\
\hline $\begin{array}{l}\text { IGT patients and normal subjects. All } \\
\text { study participants were between } 18 \text { and } \\
75 \text { years of age. } \\
\text { normal sural nerve conduction. }\end{array}$ & $\begin{array}{l}\text { Sequelae of cerebral infarction, } \\
\text { Alzheimer' s disease, Parkinson' s } \\
\text { disease, peripheral vascular disease, an } \\
\text { active foot ulcer, renal dysfunction } \\
\text { (glomerular filtration rate }[\mathrm{GFR}]<60 \\
\mathrm{~mL} / \mathrm{min} / 1.73 \mathrm{~m}^{2} \text { ), secondary causes of }\end{array}$ \\
\hline & $\begin{array}{l}\text { DPN including lumbar disease and } \\
\text { alcoholic polyneuropathy, or use of } \\
\text { drugs that could affect sweat gland } \\
\text { activity. }\end{array}$ \\
\hline
\end{tabular}

B:

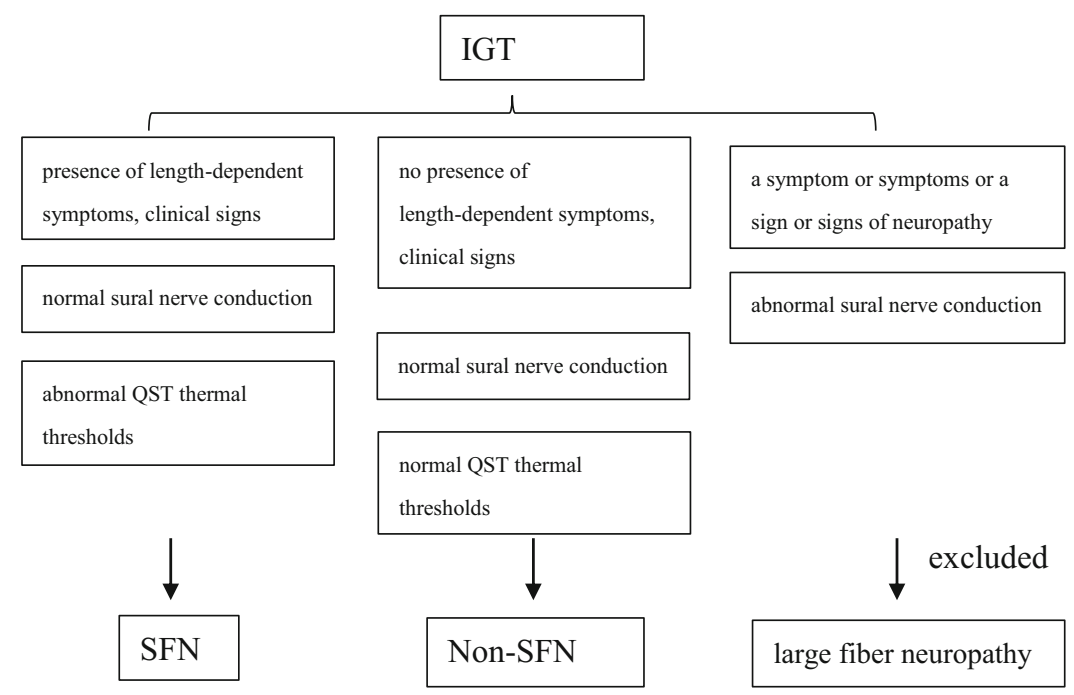

common peroneal, and posterior tibial (distal and proximal) nerves. The sensory nerve action potential (SNAP) of the sural nerve was significantly lower in the IGT-SFN group than in the CTRL and IGT-non-SFN groups (Table 4).

The average ESC (feet and hands) was significantly lower in the SFN group than in the CTRL and IGT-non-SFN groups $(p<0.05)$. The average ESC (feet and hands) in the IGT-nonSFN group was lower than that in the CTRL group, but not significantly so (Table 5).

\section{Correlation analysis}

Spearman correlation analysis indicated that the 2hPG, MNSI A, MNSI B, TCNS, and FPG levels/scores were significantly and positively

correlated with the pNF-H level $(p<0.05)$. Also, the HDL-c level, posterior tibial nerve sensory nerve conduction velocity (SNCV) (distal), sural nerve SNAP, ESC (feet), and ESC (hands) were significantly and negatively correlated with the pNF-H level $(p<0.05)$ (Table 6).

Spearman correlation analysis indicated that age, FPG, 2hPG, HbA1c, MNSI A, MNSI B, TCNS, and pNF-H levels/scores were significantly and positively correlated with SFN in IGT patients $(p<0.05)$. Also, TC and sural nerve SNAP were significantly and negatively correlated with SFN in IGT patients $(p<0.05)$ (Table 7).

After adjusting for age, BMI, HbA1c, FPG, MNSI A, MNSI B, TCNS, and sural nerve SNAP, logistical regression analysis indicated that pNFH (OR 1.029, 95\% CI 1.005, 1.054; $p=0.016)$ 
Table 2 Basic demographic and clinical data for the three groups

\begin{tabular}{|c|c|c|c|c|}
\hline \multirow[t]{2}{*}{ Parameter } & \multirow[t]{2}{*}{ CTRL $(N=33)$} & \multicolumn{2}{|l|}{ IGT $(N=44)$} & \multirow[t]{2}{*}{$p$ value } \\
\hline & & Non-SFN control & SFN & \\
\hline$N$ & 33 & 20 & 24 & \\
\hline $\mathrm{M} / \mathrm{F}$ & $10 / 23$ & $9 / 11$ & $11 / 23$ & 0.521 \\
\hline Age (years) & $50.5 \pm 8.7$ & $53.6 \pm 7.8$ & $57.7 \pm 8.8^{*}$ & 0.010 \\
\hline BMI $\left(\mathrm{kg} / \mathrm{m}^{2}\right)$ & $23.9 \pm 2.7$ & $25.22 \pm 2.53$ & $25.13 \pm 3.49$ & 0.175 \\
\hline WHR & $0.87 \pm 0.07$ & $0.9 \pm 0.07$ & $0.9 \pm 0.08$ & 0.125 \\
\hline HbAlc (\%) & $5.61 \pm 0.3$ & $5.98 \pm 0.39^{*}$ & $5.93 \pm 0.39^{*}$ & 0.002 \\
\hline FPG (mmol/l) & $5.02 \pm 0.32$ & $5.92 \pm 0.53^{*}$ & $5.89 \pm 0.53^{*}$ & $<0.001$ \\
\hline 2hPG (mmol/l) & $6.02 \pm 1.11$ & $8.87 \pm 0.75^{*}$ & $9.52 \pm 0.92^{* \#}$ & $<0.001$ \\
\hline $\mathrm{TG}(\mathrm{mmol} / \mathrm{l})$ & $1.52 \pm 0.57$ & $1.62 \pm 0.45$ & $1.60 \pm 0.99$ & 0.881 \\
\hline TC $(\mathrm{mmol} / \mathrm{l})$ & $5.59 \pm 0.73$ & $5.15 \pm 1.01$ & $5.00 \pm 0.99$ & 0.070 \\
\hline HDL-c (mmol/l) & $1.58 \pm 0.45$ & $1.51 \pm 0.35$ & $1.39 \pm 0.31$ & 0.230 \\
\hline LDL-c (mmol/l) & $3.56 \pm 0.66$ & $3.11 \pm 0.88$ & $3.10 \pm 0.96$ & 0.134 \\
\hline $\mathrm{Cr}(\mathrm{mmol} / \mathrm{l})$ & $71.47 \pm 15.25$ & $69.25 \pm 10.90$ & $70.24 \pm 17.3$ & 0.883 \\
\hline BUN (mmol/l) & $5.04 \pm 1.49$ & $5.56 \pm 0.58$ & $5.68 \pm 1.13$ & 0.132 \\
\hline $\mathrm{ACR}(\mathrm{mg} / \mathrm{mmol})$ & $0.76(0.49,1.50)$ & $1.12 \pm 0.68$ & $0.71(0.57,1.88)$ & 0.891 \\
\hline
\end{tabular}

Continuous, normally distributed variables are expressed as means \pm SD and non-normally distributed variables are expressed as medians (25\% and $75 \%$ quartiles)

$M$ male, $F$ female, $B M I$ body mass index, $W H R$ waist-to-hip ratio, $H b A 1 c$ hemoglobin A1c, FPG fasting plasma glucose, $2 h P G 2 \mathrm{~h}$ postprandial plasma glucose, $T G$ triglycerides, $T C$ total cholesterol, $H D L-c$ HDL cholesterol, $L D L-c$ LDL cholesterol, $C r$ creatinine, $B U N$ blood urea nitrogen, $A C R$ urinary albumin to urinary creatinine ratio ${ }^{*} p<0.05$ compared to CTRL, ${ }^{\#} p<0.05$ compared to IGT, based on one-way ANOVA with SNK analysis, Kruskal-Wallis $H$ test or $\chi^{2}$ test, as appropriate

Table 3 Quantitative sensory testing in control subjects and IGT patients with and without SFN

\begin{tabular}{lllll}
\hline Parameter & CTRL $(\boldsymbol{N}=\mathbf{3 3})$ & IGT $(\boldsymbol{N}=\mathbf{4 4})$ & \multirow{2}{*}{$\boldsymbol{p}$ value } \\
\cline { 2 - 4 } & & Non-SFN control (24) & SFN (20) & \\
\hline Cool threshold $\left({ }^{\circ} \mathrm{C}\right)$ & $25.64 \pm 2.96$ & $25.04 \pm 3.17$ & $24.31(22.22,26.83)^{*}$ & 0.044 \\
Warm threshold $\left({ }^{\circ} \mathrm{C}\right)$ & $39.66 \pm 2.89$ & $40.99 \pm 3.08^{*}$ & $41.55 \pm 3.13^{*}$ & 0.004 \\
Cold pain threshold $\left({ }^{\circ} \mathrm{C}\right)$ & $21.23(12.38,25.42)$ & $20.22(12.08,26.65)$ & $15.96(8.51,21.79)^{*}$ & 0.030 \\
Heat pain threshold $\left({ }^{\circ} \mathrm{C}\right)$ & $44.62 \pm 3.38$ & $45.57 \pm 3.0$ & $46.1 \pm 3.30$ & 0.056 \\
\hline
\end{tabular}

${ }^{*} p<0.05$ compared to CTRL

${ }^{\#} p<0.05$ compared to IGT-non-SFN 


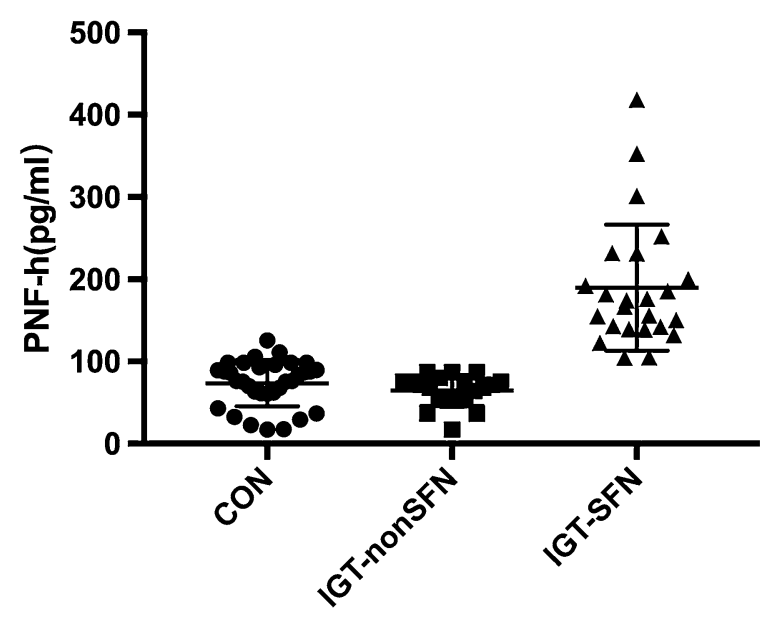

Fig. 1 Serum pNF-H levels in the CTRL, IGT-non-SFN, and IGT-SFN groups

and 2hPG (OR 2.375, 95\% CI 1.157, 4.873; $p=0.018)$ were correlated independently with SFN in the IGT patients (Table 8).

\section{DISCUSSION}

SFN is considered an early stage of DPN. SFN can be missed by physicians because of its insidious onset and difficult detection [14]. In IGT patients, SFN accounts for $42.3 \%$ of all peripheral neuropathy cases, while LFN accounts for $15.3 \%$ [4, 15]. Early identification of risk factors and specific biological markers is critical for the prevention and treatment of DPN.

Diabetic neuropathy has been linked to age and long-term hyperglycemia [16, 17], and postprandial blood glucose in particular [18]. In our study, HbA1c, FPG, and 2hPG were elevated in the IGT-SFN group, indicating that SFN is linked to blood glucose, even in prediabetes. QST did not reveal a significant difference in thermal thresholds between the IGT-SFN and IGT-non-SFN groups, but the IGT-SFN group had significantly higher thresholds than the CTRL group. Hyperglycemia may induce dysfunction of unmyelinated or thinly myelinated nerve fibers. Participants with length-dependent neurological signs and more severe thermal sensory abnormalities (as seen in our IGTSFN group) may suffer from more serious small fiber damage.

The Sudoscan results obtained in this study were similar to those attained with QST. There was no significant difference in mean ESC between the IGT-SFN and IGT-non-SFN groups. The mean ESC of the IGT-SFN group was significantly lower than that of the CTRL group. A possible explanation for this may be that many

Table 4 EMG parameters in healthy subjects and IGT patients with and without SFN

\begin{tabular}{|c|c|c|c|c|}
\hline \multirow[t]{2}{*}{ Parameter } & \multirow[t]{2}{*}{ CTRL $(N=33)$} & \multicolumn{2}{|l|}{ IGT $(N=44)$} & \multirow[t]{2}{*}{$p$ value } \\
\hline & & Non-SFN control & SFN & \\
\hline$N$ & 33 & 20 & 24 & \\
\hline Sural nerve SNCV $(\mathrm{m} / \mathrm{s})$ & $54.98 \pm 4.21$ & $55.11 \pm 5.85$ & $53.91 \pm 5.20$ & 0.464 \\
\hline Sural nerve SNAP $(\mu \mathrm{V})$ & $15.18 \pm 6.60$ & $17.45 \pm 7.56$ & $12.39 \pm 5.11^{* \#}$ & 0.004 \\
\hline Common peroneal nerve MNCV & $54.12 \pm 6.86$ & $52.94 \pm 6.02$ & $55.58 \pm 8.38$ & 0.242 \\
\hline Posterior tibial nerve MNCV & $51.03 \pm 4.75$ & $50.53 \pm 4.62$ & $52.52 \pm 7.45$ & 0.264 \\
\hline Posterior tibial nerve SNCV (distal) & $42.45 \pm 4.87$ & $40.89 \pm 8.13$ & $42.86 \pm 5.74$ & 0.337 \\
\hline Posterior tibial nerve SNCV (proximal) & $55.38 \pm 5.65$ & $58.48 \pm 7.23$ & $56.58 \pm 7.30$ & 0.132 \\
\hline Common peroneal nerve SNCV & $62.05 \pm 8.92$ & $59.94 \pm 9.74$ & $60.67 \pm 10.07$ & 0.618 \\
\hline
\end{tabular}

$S N C V$ sensory nerve conduction velocity, $M N C V$ motor nerve conduction velocity, $S N A P$ sensory nerve action potential ${ }^{*} p<0.05$ compared to CTRL

$\# p<0.05$ compared to IGT-non-SFN 
Table 5 Sudomotor function in healthy subjects and IGT patients with and without SFN

\begin{tabular}{lllll}
\hline Parameter & CTRL $(\boldsymbol{N}=33)$ & \multicolumn{2}{l}{ IGT $(\boldsymbol{N}=\mathbf{4 4})$} & \multirow{2}{*}{$\boldsymbol{p}$ value } \\
\cline { 2 - 4 } & & Non-SFN control & SFN & \\
\hline Feet ESC $(\mu S)$ & $76.79 \pm 6.63$ & $71.21 \pm 10.28$ & $69.78 \pm 14.03^{*}$ & 0.032 \\
Hand ESC $(\mu S)$ & $79.30 \pm 6.15$ & $76.58 \pm 7.74$ & $70.61 \pm 14.66^{*}$ & 0.007 \\
\hline
\end{tabular}

ESC electrochemical skin conductance

${ }^{*} p<0.05$ compared to CTRL

$\# p<0.05$ compared to IGT-non-SFN

Table 6 Spearman correlation analysis of pNF-H and clinical characteristics of the patients

\begin{tabular}{lrr}
\hline Parameter & \multicolumn{2}{c}{$\boldsymbol{R}$} \\
\hline 2hPG & $0.405^{* *}<0.001$ \\
MNSI A & $0.380^{* *}$ & 0.001 \\
MNSI B & $0.433^{* *}<0.001$ \\
TCNS & $0.495^{* *}<0.001$ \\
HDL-c & $-0.267^{*}$ & 0.021 \\
FPG & $0.229^{*}$ & 0.047 \\
Posterior tibial nerve SNCV (distal) & $-0.233^{*}$ & 0.049 \\
$\quad(\mathrm{~m} / \mathrm{s})$ & & \\
Sural nerve SNAP $(\mu \mathrm{V})$ & $-0.330^{*}$ & 0.009 \\
ESC (feet) $(\mu S)$ & $-0.314^{* *}$ & 0.006 \\
ESC (hands) $(\mu \mathrm{S})$ & $-0.352^{* *}$ & 0.002 \\
\hline
\end{tabular}

$F P G$ fasting plasma glucose, $2 h P G 2 \mathrm{~h}$ postprandial plasma glucose, $T G$ triglycerides, $T C$ total cholesterol, $H D L-c$ HDL cholesterol, ESC electrochemical skin conductance, $S N C V$ sensory nerve conduction velocity, $S N A P$ sensory nerve action potential, MNSI $A, B$ Michigan Neuropathy Screening Instrument (parts A and B), TCNS Toronto Clinical Neuropathy Score

${ }^{*} p<0.05$

${ }^{* *} p<0.01$

IGT patients with SFN do not experience autonomic neuropathy. A cross-sectional study of IGT subjects indicated that only $16 \%$ showed a loss of palmar sympathetic skin response, which indicates autonomic abnormality [19].

Axonal dysfunction can be discerned by analyzing the SNAP $[20,21]$. Our neuroelectrophysiological results indicated that the SNAP of
Table 7 Spearman correlation analysis of SFN and clinical characteristics of the patients

\begin{tabular}{lrr}
\hline Parameter & \multicolumn{1}{l}{$\boldsymbol{R}$} \\
\hline Age & $0.328^{* *}$ & \multicolumn{1}{l}{$\boldsymbol{p}$} \\
2hPG & $0.592^{* *}$ & $<0.001$ \\
FPG & $0.416^{* *}$ & $<0.001$ \\
HbAlc & $0.233^{*}$ & 0.043 \\
MNSI A & $0.290^{*}$ & 0.017 \\
MNSI B & $0.473^{* *}$ & $<0.001$ \\
TCNS & $0.695^{* *}$ & $<0.001$ \\
TC & $-0.266^{*}$ & 0.020 \\
Sural nerve SNAP (uV) & $-0.300^{*}$ & 0.011 \\
pNF-H & $0.576^{* *}$ & $<0.001$ \\
\hline
\end{tabular}

$H b A 1 c$ hemoglobin Alc, FPG fasting plasma glucose, $2 h P G 2 \mathrm{~h}$ postprandial plasma glucose, $T C$ total cholesterol, SNAP sensory nerve action potential, MNSI $A, B$ Michigan Neuropathy Screening Instrument (parts A and B), TCNS Toronto Clinical Neuropathy Score

${ }^{*} p<0.05$

${ }^{* *} p<0.01$

the sural nerve in the IGT-SFN group was statistically significantly lower than those of the other two groups, while there was no significant difference in NCV. This indicates that nerve injury during the early stage of IGT may be relatively mild and might only affect the distal sensory nerve, thus reinforcing the need to detect SFN early in IGT patients.

The levels of NSE [22], CysC [23], and TNF- $\alpha$ [24] are reported to be positively correlated with DPN. Many conditions and diseases (such as tumors, diabetic nephrology, and inflammatory 
Table 8 Multiple regression analysis of the relationship between pNF-H and SFN

\begin{tabular}{|c|c|c|c|c|c|c|c|c|}
\hline \multirow[t]{2}{*}{ Parameter } & \multirow[t]{2}{*}{$B$} & \multirow[t]{2}{*}{ S.E. } & \multirow[t]{2}{*}{ Wals } & \multirow[t]{2}{*}{ Df } & \multirow[t]{2}{*}{ Sig. } & \multirow[t]{2}{*}{ OR } & \multicolumn{2}{|c|}{ OR $(95 \% \mathrm{CI})$} \\
\hline & & & & & & & Lower & Upper \\
\hline pNF-H & 0.029 & 0.012 & 5.853 & 1 & 0.016 & 1.429 & 1.315 & 1.924 \\
\hline $2 \mathrm{hPG}$ & 0.865 & 0.367 & 5.562 & 1 & 0.018 & 2.375 & 1.157 & 4.873 \\
\hline Constant & -11.333 & 3.732 & 9.223 & 1 & 0.002 & 0.000 & & \\
\hline
\end{tabular}

$2 h P G$ 2h postprandial plasma glucose, $p N F-H$ phosphorylated neurofilament heavy chain, $B$ regression coefficient $\beta, S E$ standard error, Wals Wald Chi-Square test, $D F$ degree of freedom, Sig Significance

diseases) can affect the serum levels of these markers, and may also overlap with DPN. None of the biomarkers listed above are related to nerve structure.

Previous studies have shown that NF, one of the major axonal cytoskeletal proteins, is associated with nervous system injury [25-27]. In diabetic patients, a possible explanation for nerve injury may be impaired axonal fuction and nerve regeneration due to the accumulation of advanced glycation end products [28]. Xiaona's study [8] demonstrated higher serum pNF-H levels in patients with DPN, and that pNF-H was independently associated with DPN after adjusting for age, sex, duration, ACR, etc., but that study did not consider the association of pNF-H with small fiber neuropathy (the early stage of DPN).

Our study demonstrates that pNF-H levels in the IGT-SFN group were significantly higher than those in the CTRL and IGT-non-SFN groups. pNF-H may therefore be a sensitive indicator of SFN in IGT patients. The reason for elevated pNF-H in IGT patients is, however, unclear. Reduced expression and transportation of NFs in distal axons is one of the principal causes of axonal degeneration [29, 30]. Animal studies have demonstrated that, in STZ-induced diabetic rat models, a reduction in NFs in peripheral axons is associated with reduced myelinated nerve fiber size [31]. Small fiber nerves are unmyelinated or thinly myelinated, and are easily damaged under hyperglycemic conditions and by inflammatory factors. This may explain the elevated pNF-H seen in our SFN patients.

The correlations of neuropathy assessment measures (MNSI A, MNSI B, TCNS) with SFN observed in this study indicate that length-dependent symptoms and signs are features of SFN. The negative correlation between TC and SFN indicates that TC may protect against SFN. Appropriate cholesterol levels promote the formation of nerve fibers and help maintain the function of neurons [32, 33].

$2 \mathrm{hPG}$ was an independent predictor of SFN in this study. Kannan found that 2hPG was an independent risk factor for sensory nerve dysfunction, which is in good accord with the results of this study [34].

As it is a cross-sectional study, this study could not determine the cause and effect relationship between elevated pNF-H and SFN. Also, the study sample was relatively small due to poor compliance of the IGT patients and the complexity of the tests. A prospective trial including a larger population is planned to investigate the influence of additional factors on SFN, and to study the efficacy of early intervention in the treatment of SFN patients.

\section{CONCLUSION}

Our study revealed that serum pNF-H may be associated with SFN in IGT patients, even after adjusting for age, BMI, HbA1c, FPG, MNSI A, MNSI B, TCNS, and sural nerve SNAP. Serum pNF-H may therefore be a potential biomarker for the detection of SFN.

The major limitation of the present study is the small number of participants. Another limitation is that it is a cross-sectional study, meaning that it could not be used to determine the cause and effect relationship between elevated pNF-H and SFN. We expect that the results of our planned prospective study, which 
will include a larger population, will support the results of the present study.

\section{ACKNOWLEDGEMENTS}

We thank the participants in the study. The authors would also like to thank Sun Yu, Liu Lele, Zhang Jianshuan, and Chen Ying for technical assistance, and Li Daiqing for helpful ideas and support.

Funding. This work was supported by a Tianjin Medical University Science Grant (General Program; 2016KYZM04), a Tianjin Science Grant (Youth Program; 18JCQNJC79900), and Scientific Research Funding of Tianjin Medical University Chu Hsien-I Memorial Hospital 2016DX05. The journal's Rapid Service Fee was funded by the authors.

Authorship. All named authors meet the International Committee of Medical Journal Editors (ICMJE) criteria for authorship for this article, take responsibility for the integrity of the work as a whole, and have given their approval for this version to be published.

Authorship Contributions. Involved in study design and conduct: YpL and YX; assessment of general conditions: AlY and JyX; data collection, management, analysis: $\mathrm{YpL}$ and $\mathrm{ZqY}$; interpretation: YrZ, LZ, and $\mathrm{SH}$; manuscript preparation, review, or approval: YpL and YX.

Disclosures. Yu-peng Li, Zhong-qing Yan, Li-ping Han, Ai-li Yin, Jin-yong Xu, Ya-ran Zhai, Sai Hao, Lin Zhang, and Yun Xie have nothing to disclose.

Compliance with Ethics Guidelines. This research was conducted in accordance with the Declaration of Helsinki. The study protocol was approved by the Ethics Committee of Tianjin Medical University Metabolic Diseases Hospital. All study participants provided informed consent.

Data Availability. Requests for access to the study data can be submitted via email to dr_liyupeng@126.com.
Open Access. This article is distributed under the terms of the Creative Commons Attribution-NonCommercial 4.0 International License (http://creativecommons.org/licenses/ by-nc/4.0/), which permits any noncommercial use, distribution, and reproduction in any medium, provided you give appropriate credit to the original author(s) and the source, provide a link to the Creative Commons license, and indicate if changes were made.

\section{REFERENCES}

1. Boulton AJ, Vinik AI, Arezzo JC, et al. Diabetic neuropathies: a statement by the American Diabetes Association. Diabetes Care. 2005;28:956-62.

2. Said G. Diabetic neuropathy: a review. Nat Clin Pract Neurol. 2007;3:331-40.

3. Ragé M, Van Acker N, Knaapen MW, et al. Asymptomatic small fiber neuropathy in diabetes mellitus: investigations with intraepidermal nerve fiber density, quantitative sensory testing and laser-evoked potentials. J Neurol. 2011;258:1852-64.

4. Green AQ, Krishnan S, Finucane FM, Rayman G. Altered C-fiber function as an indicator of early peripheral neuropathy in individuals with impaired glucose tolerance. Diabetes Care. 2010;33:174-6.

5. Cappellari A, Airaghi L, Capra R, et al. Early peripheral nerve abnormalities in impaired glucose tolerance. Electromyogr Clin Neurophysiol. 2005;45:241-4.

6. Cortez M, Singleton JR, Smith AG. Glucose intolerance, metabolic syndrome, and neuropathy. Handb Clin Neurol. 2014;126:109-22.

7. Vas PR, Sharma S, Rayman G. Distal sensorimotor neuropathy: improvements in diagnosis. Rev Diabet Stud. 2015;12:29-47.

8. Qiao X, Zhang S, Zhao W, et al. Serum phosphorylated neurofilament-heavy chain, a potential biomarker, is associated with peripheral neuropathy in patients with type 2 diabetes. Medicine (Baltimore). 2015;94:e1908.

9. Dori A, Lopate G, Keeling R, Pestronk A. Myovascular innervation: axon loss in small-fiber neuropathies. Muscle Nerve. 2015;51:514-21.

10. Buchmann SJ, Penzlin AI, Kubasch ML, Illigens BM, Siepmann T. Assessment of sudomotor function. Clin Auton Res. 2019;29:41-53. 
11. Casanova-Molla J, Morales M, Garrabou G, et al. Mitochondrial loss indicates early axonal damage in small fiber neuropathies. J Peripher Nerv Syst. 2012;17:147-57.

12. Feldman EL, Stevens MJ, Thomas PK, Brown MB, Canal N, Greene DA. A practical two-step quantitative clinical and electrophysiological assessment for the diagnosis and staging of diabetic neuropathy. Diabetes Care. 1994;17:1281-9.

13. Tesfaye S, Boulton AJ, Dyck PJ, et al. Diabetic neuropathies: update on definitions, diagnostic criteria, estimation of severity, and treatments. Diabetes Care. 2010;33:2285-93.

14. Hoeijmakers JG, Faber CG, Merkies IS, Waxman SG. Painful peripheral neuropathy and sodium channel mutations. Neurosci Lett. 2015;596:51-9.

15. Sumner CJ, Sheth S, Griffin JW, Cornblath DR, Polydefkis $M$. The spectrum of neuropathy in diabetes and impaired glucose tolerance. Neurology. 2003;60:108-11.

16. Li L, Chen J, Wang J, Cai D. Prevalence and risk factors of diabetic peripheral neuropathy in type 2 diabetes mellitus patients with overweight/obese in Guangdong province, China. Prim Care Diabetes. 2015;9:191-5.

17. Jaiswal M, Divers J, Dabelea D, et al. Prevalence of and risk factors for diabetic peripheral neuropathy in youth with type 1 and type 2 diabetes: search for diabetes in youth study. Diabetes Care. 2017;40:1226-32.

18. $\mathrm{Lu} \mathrm{B}, \mathrm{Hu}$ J, Wen J, et al. Determination of peripheral neuropathy prevalence and associated factors in Chinese subjects with diabetes and pre-diabetesShangHai Diabetic Neuropathy Epidemiology and Molecular Genetics Study (SH-DREAMS). PLoS One. 2013;8:e61053.

19. Tiftikcioglu BI, Bilgin S, Duksal T, Kose S, Zorlu Y. Autonomic neuropathy and endothelial dysfunction in patients with impaired glucose tolerance or type 2 diabetes mellitus. Medicine (Baltimore). 2016;95:e3340.

20. Roberts NM, Wertsch JJ. Measuring sensory nerve action potential electrical power. Muscle Nerve. 2010;41:318-23.

21. Krajewski KM, Lewis RA, Fuerst DR, et al. Neurological dysfunction and axonal degeneration in Charcot-Marie-Tooth disease type 1A. Brain. 2000;123(Pt 7):1516-27.

22. Li J, Zhang H, Xie M, Yan L, Chen J, Wang H. NSE, a potential biomarker, is closely connected to diabetic peripheral neuropathy. Diabetes Care. 2013;36:3405-10.

23. $\mathrm{Hu} \mathrm{Y,} \mathrm{Liu} \mathrm{F,} \mathrm{Shen} \mathrm{J,} \mathrm{et} \mathrm{al.} \mathrm{Association} \mathrm{between}$ serum cystatin $C$ and diabetic peripheral neuropathy: a cross-sectional study of a Chinese type 2 diabetic population. Eur J Endocrinol. 2014;171:641-8.

24. Li X, Zhu J, Liu N, Liu J, Zhang Z. TNF-alpha in peripheral neuropathy patients with impaired glucose regulation. J Diabetes Res. 2017;2017:7024024.

25. Shaw G, Yang C, Ellis R, et al. Hyperphosphorylated neurofilament NF-H is a serum biomarker of axonal injury. Biochem Biophys Res Commun. 2005;336:1268-77.

26. Su W, Chen HB, Li SH, Wu DY. Correlational study of the serum levels of the glial fibrillary acidic protein and neurofilament proteins in Parkinson's disease patients. Clin Neurol Neurosurg. 2012;114:372-5.

27. Singh P, Yan J, Hull R, et al. Levels of phosphorylated axonal neurofilament subunit $\mathrm{H}(\mathrm{pNfH})$ are increased in acute ischemic stroke. J Neurol Sci. 2011;304:117-21.

28. Sugimoto K, Yasujima M, Yagihashi S. Role of advanced glycation end products in diabetic neuropathy. Curr Pharm Des. 2008;14:953-61.

29. Fernyhough P, Schmidt RE. Neurofilaments in diabetic neuropathy. Int Rev Neurobiol. 2002;50:115-44.

30. Fernyhough P, Gallagher A, Averill SA, et al. Aberrant neurofilament phosphorylation in sensory neurons of rats with diabetic neuropathy. Diabetes. 1999;48:881-9.

31. Yagihashi S, Kamijo M, Watanabe K. Reduced myelinated fiber size correlates with loss of axonal neurofilaments in peripheral nerve of chronically streptozotocin diabetic rats. Am J Pathol. 1990;136:1365-73.

32. Pfrieger FW, Ungerer N. Cholesterol metabolism in neurons and astrocytes. Prog Lipid Res. 2011;50:357-71.

33. Fan QW, Yu W, Gong JS, et al. Cholesterol-dependent modulation of dendrite outgrowth and microtubule stability in cultured neurons. J Neurochem. 2002;80:178-90.

34. Németh N, Putz Z, Istenes I, et al. Is there a connection between postprandial hyperglycemia and IGT related sensory nerve dysfunction. Nutr Metab Cardiovasc Dis. 2017;27:609-14. 